\title{
Interactive Effects of Two Soilborne Pathogens, Phytophthora capsici and Verticillium dahliae, on Chile Pepper
}

\author{
S. Sanogo
}

Department of Entomology, Plant Pathology, and Weed Science, New Mexico State University, Las Cruces 88003. Accepted for publication 10 July 2006.

\begin{abstract}
Sanogo, S. 2007. Interactive effects of two soilborne pathogens, Phytophthora capsici and Verticillium dahliae, on chile pepper. Phytopathology 97:37-43.

Phytophthora capsici and Verticillium dahliae are two mycelial microorganisms associated with wilt symptoms on chile pepper (Capsicum annuum). Both pathogens occur in the same field and can infect a single plant. This study examined the nature of the co-occurrence of $P$. capsici and $V$. dahliae. Chile pepper plants were inoculated with each pathogen separately or with both pathogens concomitantly or sequentially. In concomitant inoculations, plants were inoculated with a mixture of zoospores of $P$. capsici and conidia of $V$. dahliae. In sequential inoculations, plants were inoculated with zoospores of $P$. capsici 4 days prior to inoculation with conidia of $V$. dahliae, or plants were inoculated with conidia of $V$. dahliae 4 days prior to inoculation with zoospores of $P$. capsici. Stem necrosis and leaf wilting were visible 3 to 4 days earlier in plants inoculated with both $P$. capsici and $V$. dahliae than in plants

served in plants inoculated with both $P$. capsici and $V$. dahliae by 21 days after inoculation. These symptoms were not observed in control plants or plants inoculated with $V$. dahliae alone. The frequency of recovery of $V$. dahliae from stems was $\approx 85$ to $140 \%$ higher across inoculum levels when plants were inoculated with both $P$. capsici and $V$. dahliae than when plants were inoculated by $V$. dahliae alone. Similarly, the frequency of recovery of $V$. dahliae from roots was $\approx 13$ to $40 \%$ higher across inoculum levels when plants were inoculated with both $P$. capsici and $V$. dahliae than when plants were inoculated by $V$. dahliae alone. There was no apparent antagonism between the two pathogens when they were paired on growth media. In general, when $P$. capsici and $V$. dahliae were paired on growth media, mycelial growth of each pathogen grown alone was not significantly different from mycelial growth when the pathogens were paired. Results suggest that wilt development is hastened by the presence of both $P$. capsici and $V$. dahliae in the same plants. The presence of $P$. capsici and $V$. dahliae in the same inoculum court enhanced infection and colonization of chile pepper by $V$. dahliae.
\end{abstract} inoculated with $P$. capsici alone. Stem necrosis and generalized plant wilting were observed in plants inoculated with $P$. capsici alone, and stem necrosis, generalized plant wilting, and vascular discoloration were ob-
Additional keywords: coexistence, co-infection, Verticillium wilt.
Phytophthora blight caused by Phytophthora capsici, and Verticillium wilt caused by Verticillium dahliae, are two diseases which constitute a limiting factor to profitable production of many crops $(6,14)$ in the United States and worldwide. Phytophthora blight and Verticillium wilt are the most prevalent diseases on chile pepper (Capsicum annuum) in New Mexico (17) and, on this crop, $P$. capsici and $V$. dahliae display differential infection patterns (16). P. capsici causes root rot and blight of stems and fruit. Infection of the root and lower portion of the stem leads to plant wilting, which is the most conspicuous symptom of the disease. $V$. dahliae invades roots without causing rotting, and colonizes the vascular tissues in roots and stems. Visual symptoms of plant infection by $V$. dahliae include stunting, defoliation, and wilting, with necrosis of vascular tissues.

Phytophthora blight and Verticillium wilt may severely affect physiologic processes in pepper plants and reduce crop yield. In plants inoculated with $P$. capsici, $\mathrm{CO}_{2}$ exchange and leaf conductance were reduced by $\approx 74 \% 4$ days after inoculation (1). Xie et al. (21) reported that Phytophthora blight decreased yield of green chile pepper by $55 \%$ and combined yield of green and red chile pepper by $36 \%$. Similarly, Biles et al. (2) reported that yield of green chile pepper was reduced by 48 to $55 \%$ in fields infested by P. capsici.

Although the impact of Verticillium wilt on chile pepper yield has not been quantified, several physiological processes such as

Corresponding author: S. Sanogo; E-mail address: ssanogo@nmsu.edu

DOI: $10.1094 /$ PHYTO-97-0037

C 2007 The American Phytopathological Society respiration, transpiration, and photosynthesis are affected in plants infected with $V$. dahliae and, therefore, yield reduction may be incurred $(9,10,19)$. For example, in pepper plants, $\mathrm{CO}_{2}$ uptake was reduced by 34 and $50 \%$ at 40 days and 95 days, respectively, after inoculation with $V$. dahliae (19). Infection of pepper plants by $V$. dahliae reduced photosynthesis through defoliation and chlorophyll degradation (10), and led to a decrease in leaf water potential along with a buildup of proline and total sugars, and a pronounced decrease in starch and protein levels in leaves and concentration of abscisic acid in xylem (9).

Plants infected with $P$. capsici and $V$. dahliae are found in the same field, and both pathogens also can infect a single plant (17). In a 3-year etiological survey of wilt symptoms in 59 chile pepper fields (17), plants infected with $V$. dahliae and $P$. capsici were found in 80 and $100 \%$ of the fields, respectively, and the two pathogens were found in $80 \%$ of the fields, and occurred together in some wilted plants in $12 \%$ of the fields. The occurrence of both pathogens within a single plant has raised the question about their interactive effects on plant infection. In a previous study (17), it was postulated that co-infection may hasten the development of plant wilt if $V$. dahliae and $P$. capsici interact in a synergistic fashion. Little is known about the nature of these pathogenic interactions. An increased understanding of the nature of interactions between $P$. capsici and $V$. dahliae may help elucidate ecological factors affecting these pathogens, and may have direct application in designing an effective control strategy for wilt on chile pepper.

The objective of this study was to evaluate the effects of interspecific interactions between $P$. capsici and $V$. dahliae on infection of chile pepper and on their respective vegetative growth. 


\section{MATERIALS AND METHODS}

Maintenance of cultures and inoculum preparation. An isolate of $V$. dahliae and isolate PWB-24 of P. capsici (3) recovered from a naturally field-infected chile pepper plant in New Mexico were used in this study. These two pathogenic isolates were maintained on water agar in 9-cm-diameter plastic petri plates. Inoculum used in plant infection experiments consisted of conidia of $V$. dahliae and zoospores of $P$. capsici. To produce conidia, a 1-cm mycelial plug of $V$. dahliae grown on water agar was added to $100 \mathrm{ml}$ of sterilized Czapek-Dox broth contained in a 250-ml Erlenmeyer flask. All flasks were placed on a rotary shaker and incubated at room temperature $\left(23\right.$ to $\left.25^{\circ} \mathrm{C}\right)$ in darkness for 7 to 10 days. Then, the contents of the flask were passed through three layers of cheesecloth to separate conidia from mycelial plugs and mats. The number of conidia in the filtered suspension was estimated with a hemacytometer and adjusted to $4 \times 10^{6}$ conidia/ml .

Production of zoospores of $P$. capsici was performed as follows. A $1-\mathrm{cm}$ mycelial plug of $P$. capsici grown on water agar was placed onto a 9-cm-diameter petri plate containing V8 juice agar and incubated in the dark at $25^{\circ} \mathrm{C}$. After 5 days, six mycelial plugs $(1 \mathrm{~cm}$ in diameter) were cut from the V8 juice agar culture. The plugs were placed in $20 \mathrm{ml}$ of sterile distilled water in a 9-cm-diameter petri plate and maintained in the dark at $25^{\circ} \mathrm{C}$ for 3 days to induce sporangia formation. The plates then were transferred to $10^{\circ} \mathrm{C}$ for $60 \mathrm{~min}$ and returned to $25^{\circ} \mathrm{C}$ for $90 \mathrm{~min}$ to trigger the release of zoospores. The contents of petri plates then were passed through three layers of cheesecloth to remove agar plugs. The number of zoospores was counted using a hemacytometer, and inoculum concentration was adjusted to $2 \times$ $10^{3}$ zoospores perml.

Plant production and inoculation. Seed of the chile pepper cv. Joe Parker, which is known to be susceptible to both $P$. capsici and $V$. dahliae (3), were planted in sterilized vermiculite in 6-cell flats in the greenhouse. At the first true leaf stage, seedlings were transplanted individually into $12-\mathrm{cm}$ round plastic pots filled with a sterilized mixture of Terra-Lite Metro Mix 360 (W. R. Grace \& Co., Memphis, TN), sand, and perlite (2:1:1 per volume). Seedlings were fertilized once a week by adding to each pot a $150-\mathrm{ml}$ solution of $20 \% \mathrm{~N}, 20 \% \mathrm{P}_{2} \mathrm{O}_{5}$, and $20 \% \mathrm{~K}_{2} \mathrm{O}$ fertilizer (Scotts Co., Marysville, $\mathrm{OH}$ ) containing $3.94 \%$ ammoniacal nitrogen, $6.05 \%$ nitrate, $10.01 \%$ urea, $0.05 \% \mathrm{Mg}, 0.0068 \% \mathrm{~B}, 0.0036 \% \mathrm{Cu}$ (chelated $\mathrm{Cu}$ ), $0.05 \% \mathrm{Fe}$ (chelated Fe), $0.025 \% \mathrm{Mn}$ (chelated $\mathrm{Mn}$ ), $0.0009 \% \mathrm{Mo}$, and $0.0025 \% \mathrm{Zn}$ (chelated $\mathrm{Zn}$ ). Plants were inoculated by dispensing 5 or $10 \mathrm{ml}$ of inoculum suspension on top of the soil in each pot around the base of each plant. The addition of 5 or $10 \mathrm{ml}$ of conidial suspension of $V$. dahliae generated two inoculum levels of $2 \times 10^{7}$ (low level, V1) and $4 \times$ $10^{7}$ (high level, V2) conidia per pot, respectively. Similarly, the addition of 5 or $10 \mathrm{ml}$ of zoospore suspension of $P$. capsici yielded two inoculum levels of $1 \times 10^{4}$ (low level, P1) and $2 \times 10^{4}$ (high level, P2) zoospores per pot, respectively. For seedlings serving as control (noninoculated), no inoculum suspension was added to the soil in each pot.

Simultaneous and sequential inoculations. Plant production, inoculum production, and plant inoculation were done as described above. Two sets of experiments were conducted. In the first set, seedlings at the one- to four-flower-bud stage were inoculated with $V$. dahliae, $P$. capsici, and a mixture of both pathogens. There were nine treatments consisting of two inoculum levels of $P$. capsici $(\mathrm{P} 1=$ low and $\mathrm{P} 2=$ high, as described above) and two inoculum levels of $V$. dahliae (V1 = low and V2 = high, as described above), four combinations of both pathogens at each of the two inoculum levels (P1V1, P1V2, P2V1, and P2V2), and a control treatment (noninoculated). The nine treatments were evaluated in a randomized complete block design on a greenhouse bench. Each treatment was replicated six times, with a pot being a replicate. Experiments were terminated 21 days after inoculation and were completed three times.

In the second set of experiments, treatments were identical to those in the first set of experiments described above, except that plants were inoculated with $V$. dahliae 4 days prior to being inoculated with $P$. capsici, or plants were inoculated with $P$. capsici 4 days prior to being inoculated with $V$. dahliae. Overall, 13 treatments were established consisting of two inoculum levels of $P$. capsici ( $\mathrm{P} 1=$ low and $\mathrm{P} 2=$ high), two inoculum levels of $V$. dahliae ( $\mathrm{V} 1=$ low and $\mathrm{V} 2=$ high), eight combinations of both pathogens at each of the two inoculum levels (P1BV1, $\mathrm{P} 1 \mathrm{BV} 2, \mathrm{P} 2 \mathrm{BV} 1, \mathrm{P} 2 \mathrm{BV} 2, \mathrm{~V} 1 \mathrm{BP} 1, \mathrm{~V} 2 \mathrm{BP} 1, \mathrm{~V} 1 \mathrm{BP} 2$, and V2BP2), and a control (C) treatment (noninoculated). The letter "B" signifies "before" and indicates which pathogen and inoculum level were used first in the sequential inoculations. For example, inoculation of plants with $P$. capsici at low inoculum level (P1) 4 days before inoculation with $V$. dahliae at low inoculum level (V1) is denoted as P1BV1. Similarly, inoculation of plants with $V$. dahliae at low inoculum level (V1) 4 days before inoculation with $P$. capsici at low inoculum level (P1) is denoted as V1BP1. Each treatment was replicated six times, with a pot being a replicate. The experiments were terminated 21 days after inoculation with $P$. capsici and were completed twice.

Assessment of plant infection. In both sets of experiments, plant infection was assessed in the following two ways: (i) severity of disease symptoms on aboveground parts and (ii) plant colonization. Three plants were allocated for assessment of disease severity, and the other three plants were used to gauge plant colonization. Severity of disease symptoms on aboveground plant parts was evaluated using a scale as follows: $0=$ no visual disease symptom, 1 = vascular discoloration or stem necrosis, $2=$ vascular discoloration and stem necrosis, $3=$ wilting and no vascular discoloration, $4=$ wilting and vascular discoloration, and $5=$ dead. Vascular discoloration was assessed by sectioning each plant longitudinally. Presence or absence of vascular discoloration was recorded.

To evaluate plant colonization by the pathogens, three plants were uprooted from each treatment, and a piece of tap root at least $6 \mathrm{~cm}$ long was recovered. Each plant was washed free of soil and secondary roots and leaves were removed. The plant was surface sterilized by submersion in $0.5 \%$ sodium hypochlorite in a $250-\mathrm{ml}$ graduated cylinder for $2 \mathrm{~min}$. The plant was rinsed in sterile distilled water, dried between two layers of sterile paper towels, and cut at the crown to separate root and stem parts. Each part was sectioned into $1.5-\mathrm{cm}$ segments with a surgical blade, which was dipped in 95\% ethyl alcohol and flame sterilized between segments. Each segment was split longitudinally, yielding two sections, which were plated onto $2 \%$ water agar medium $(20 \mathrm{~g}$ of agar per liter of distilled water) in 9-cm-diameter plates with the cut surface on the medium. The segments were examined for $P$. capsici or $V$. dahliae after 10 to 14 days of incubation at $25^{\circ} \mathrm{C}$. Percentage of root and stem segments yielding P. capsici alone, $V$. dahliae alone, or both microorganisms was recorded.

Pathogen interaction on growth media. Mycelial growth of $V$. dahliae and P. capsici was evaluated on four solid media: $10 \%$ V8 juice agar (100 $\mathrm{ml}$ of clarified V8 juice, $20 \mathrm{~g}$ of agar, and $900 \mathrm{ml}$ of distilled water), potato dextrose agar (39 g per liter of distilled water), $2 \%$ water agar (20 g of agar per liter of distilled water), and Czapek-Dox agar (49 g per liter of distilled water). On each solid medium, $V$. dahliae and $P$. capsici were grown separately or paired by placing a $1-\mathrm{cm}$ plug of each isolate $2 \mathrm{~cm}$ apart. Two sets of experiments were conducted. In the first set of experiments, $V$. dahliae and $P$. capsici were placed concomitantly on each medium for $72 \mathrm{~h}$ or 7 days, after which radii of colonies were measured. In the second set of experiments, $V$. dahliae was placed on each medium 7 days before being paired with $P$. capsici, and colony radii were measured $72 \mathrm{~h}$ after pairing of the isolates. In both sets of experiment, all plates were placed in a 
plastic box and incubated in darkness at $25^{\circ} \mathrm{C}$. In each experiment, radius of mycelial growth from plugs was measured from the edge of the plug to the margin of mycelial growth. Three radius measurements were made on mycelial growth from each plug and averaged per plate. Each set of experiments was conducted in a completely randomized design, with five to six replications per treatment and a petri plate as a replicate. Each experiment was completed at least three times.

To test whether volatile substances were produced and involved in the outcome of the interaction between $P$. capsici and $V$. dahliae, plates with the four solid media described above (V8 juice agar, potato dextrose agar, water agar, and Czapek-Dox agar) were used. Plates containing $V$. dahliae were inverted and placed over plates containing $P$. capsici. Similarly, plates containing $P$. capsici were inverted and placed over plates containing $V$. dahliae. The two plates were secured in place with parafilm which was overlaid with two layers of white masking tape. All plates were maintained in darkness at $25^{\circ} \mathrm{C}$. Radius of mycelial growth was measured as described above after 7 days of incubation. The experiment was conducted in a completely randomized design with six replications, with a petri plate as a replicate. The experiment was conducted three times.

Data analysis. The variables measured in this study consist of (i) disease severity, (ii) percentage of root and stem colonization, (iii) and radius of mycelial growth. Data collected on radius of mycelial growth and percentage of root and stem colonization were analyzed by analysis of variance using the PROC MIXED procedure in SAS (SAS Institute, Cary, NC). The data consisting of disease severity ratings were ordinal; therefore, these data were analyzed by nonparametric approaches using SAS macros OWL and LD_CI as outlined by Brunner et al. (4). These macros allow computation of mean rank of treatments, relative treatment effects (RTE), 95\% confidence intervals for RTE, and Kruskal-Wallis test statistic $\left(\mathrm{Q}_{\mathrm{n}}\right)$ for gauging the significance of treatments. The $P$ value associated with the Kruskal-Wallis test statistic $\left(Q_{n}\right)$ is determined using $\chi^{2}$ distribution. Data from individual experiments were analyzed separately to determine consistency of results and homogeneity of error variance among repetitions of experiments. Error variances were evaluated for homogeneity using procedures outlined by Gomez and Gomez (11). When results were consistent among experiments and the error variances were homogeneous $(P>0.05)$, results from all repetitions of experiments were combined. Arcsine square root transformation was applied to data consisting of percentage (converted to proportion) of root and stem colonization before analysis of variance. All statistical tests were assessed at 5\% significance level, and treatment differences were gauged on $95 \%$ confidence intervals for RTE and preplanned linear contrasts among treatments for tissue colonization and mycelial growth data.

\section{RESULTS}

Simultaneous inoculations. Stem necrosis and leaf wilting were apparent in plants inoculated simultaneously with $P$. capsici and $V$. dahliae within 7 to 10 days, and in plants inoculated with $P$. capsici alone within 10 to 14 days after inoculation. These symptoms were not observed in control plants or plants inoculated with $V$. dahliae alone. By 21 days after inoculation, stem necrosis and generalized plant wilting were observed in plants inoculated with $P$. capsici alone, and stem necrosis, generalized plant wilting, and vascular discoloration were observed in plants inoculated with both $P$. capsici and $V$. dahliae. Whereas vascular discoloration, which is associated with infection by $V$. dahliae, was observed in plants inoculated with both $P$. capsici and $V$. dahliae, this symptom was not observed in plants inoculated with $V$. dahliae alone. Control plants did not display any stem necrosis, vascular discoloration, or wilting. There was a significant difference among treatments $\left(P<10^{-8}\right)$ as determined by
Kruskal-Wallis test (Table 1). The RTE for the two inoculum levels of $V$. dahliae used alone (V1 and V2) were identical to that of the control treatment, with an RTE $=0.166$ (Table 1). In contrast, in the presence of $P$. capsici, RTE values were 0.623 and 0.656 at low (V1) and high (V2) inoculum levels of $V$. dahliae, respectively, when $P$. capsici was used at a low level (P1). Similarly, RTE values were 0.830 and 0.860 at low (V1) and high (V2) inoculum levels of $V$. dahliae, respectively, when $P$. capsici was used at a high level (P2). As indicated by the overlapping 95\% confidence intervals, the RTE were similar between the two inoculum levels of $V$. dahliae (V1 and V2) at each inoculum level of $P$. capsici (P1 or $\mathrm{P} 2)$.

Frequency of recovery of $P$. capsici from stems of plants inoculated by $P$. capsici alone was $\approx 19$ and $29 \%$ at low (P1) and high (P2) levels of inoculum of $P$. capsici, respectively (Fig. 1A). In plants inoculated with both $P$. capsici and $V$. dahliae, $P$. capsici never was recovered alone, but always was recovered along with $V$. dahliae, and the levels of recovery were comparable with those from plants inoculated by $P$. capsici alone. Concomitant recovery of $P$. capsici and $V$. dahliae from stems was restricted to segments with necrosis in the lower stem area. The frequencies of isolation of both pathogens ranged from 17 to $21 \%$ across the combination of inoculum levels (P1V1, P1V2, P2V1, and P2V2) of both pathogens, and there was no significant difference among these treatments as assessed by linear contrasts among treatments $(P>$ $0.10)$.

Frequency of isolation of $V$. dahliae from stem segments was $\approx 37$ and $45 \%$ in plants inoculated with $V$. dahliae alone at low (V1) and high (V2) inoculum levels of $V$. dahliae, respectively (Fig. 1A). In contrast, in the presence of $P$. capsici used at a low inoculum level (P1), frequency of isolation of $V$. dahliae was increased to $\approx 57$ and $65 \%$ at low (V1) and high (V2) inoculum levels of $V$. dahliae, respectively. The frequency of isolation of $V$. dahliae was further augmented to 73 and $83 \%$ at low (V1) and high (V2) inoculum levels of $V$. dahliae, respectively, when $P$. capsici was used at a high inoculum level (P2). These frequencies of recovery of $V$. dahliae from stem segments in the presence of $P$. capsici were significantly higher, by 43 to $97 \%$, than the frequency of its recovery when plants were inoculated with $V$. dahliae alone, as revealed by linear contrasts $(P<$ 0.0001). Taking into account the levels of concomitant recovery (stem segments with both $V$. dahliae and $P$. capsici), the frequency of recovery of $V$. dahliae was higher, by 85 to $140 \%$, than the frequency of its recovery when plants were inoculated with V. dahliae alone.

TABLE 1. Median, mean rank, relative treatment effects (RTE), and 95\% confidence intervals (CI) for RTE of severity ratings on chile pepper (cv. Joe Parker) inoculated simultaneously with Phytophthora capsici or Verticillium dahliae $^{\mathrm{a}}$

\begin{tabular}{lcccc}
\hline Treatment $^{\mathrm{b}}$ & Median $^{\mathrm{c}}$ & Mean rank & RTE & 95\% CI for RTE \\
\hline C & 0 & 14 & 0.166 & $(0.166,0.166)$ \\
P1 & 1 & 39.22 & 0.478 & $(0.413,0.543)$ \\
P1V1 & 3 & 51 & 0.623 & $(0.517,0.730)$ \\
P1V2 & 3 & 53.66 & 0.656 & $(0.564,0.750)$ \\
P2 & 3 & 45.38 & 0.554 & $(0.490,0.618)$ \\
P2V1 & 4 & 67.72 & 0.830 & $(0.780,0.881)$ \\
P2V2 & 4 & 70 & 0.860 & $(0.830,0.886)$ \\
V1 & 0 & 14 & 0.166 & $(0.166,0.166)$ \\
V2 & 0 & 14 & 0.166 & $(0.166,0.166)$ \\
\hline
\end{tabular}

a Kruskal-Wallis test: test statistic $=70.51, P<10^{-8}$.

b Treatments consisted of two inoculum levels of $P$. capsici $(\mathrm{P} 1=$ low and $\mathrm{P} 2$ $=$ high), two inoculum levels of $V$. dahliae $(\mathrm{V} 1=$ low and $\mathrm{V} 2=$ high$)$, four combinations of both pathogens at each of the two inoculum levels (P1V1, P1V2, P2V1, and P2V2), and a control (C) treatment (noninoculated).

${ }^{c}$ Disease severity was rated using the following scale: $0=$ no visual disease symptom, 1 = vascular discoloration or stem necrosis, 2 = vascular discoloration and stem necrosis, 3 = wilting and no vascular discoloration, $4=$ wilting and vascular discoloration, and $5=$ dead. 
In plants inoculated with $P$. capsici alone, all root segments yielded $P$. capsici (Fig. 1B). In plants inoculated with $V$. dahliae alone, frequency of isolation of $V$. dahliae from roots was $\approx 47$ and $69 \%$ at the low (V1) and high (V2) inoculum levels of V. dahliae, respectively. In simultaneous inoculation with both pathogens, the frequency of root segments yielding $P$. capsici alone ranged from $\approx 3$ to $47 \%$, whereas no root segments yielded $V$. dahliae alone. However, both pathogens were recovered from root segments at frequencies ranging from $\approx 53$ to $97 \%$. Hence, simultaneous inoculation of plants with both pathogens resulted in greater percentage of root colonization by $V$. dahliae than when plants were inoculated by $V$. dahliae alone.

Sequential inoculations. Stem necrosis and leaf wilting were visible 3 to 4 days earlier in plants inoculated with both $P$. capsici and $V$. dahliae than in plants inoculated with $P$. capsici alone. Stem necrosis and generalized plant wilting were observed in plants inoculated with $P$. capsici alone, and stem necrosis, generalized plant wilting, and vascular discoloration were observed in plants inoculated with both $P$. capsici and $V$. dahliae by 21 days after inoculation. These symptoms were not observed in control plants or plants inoculated with $V$. dahliae alone.
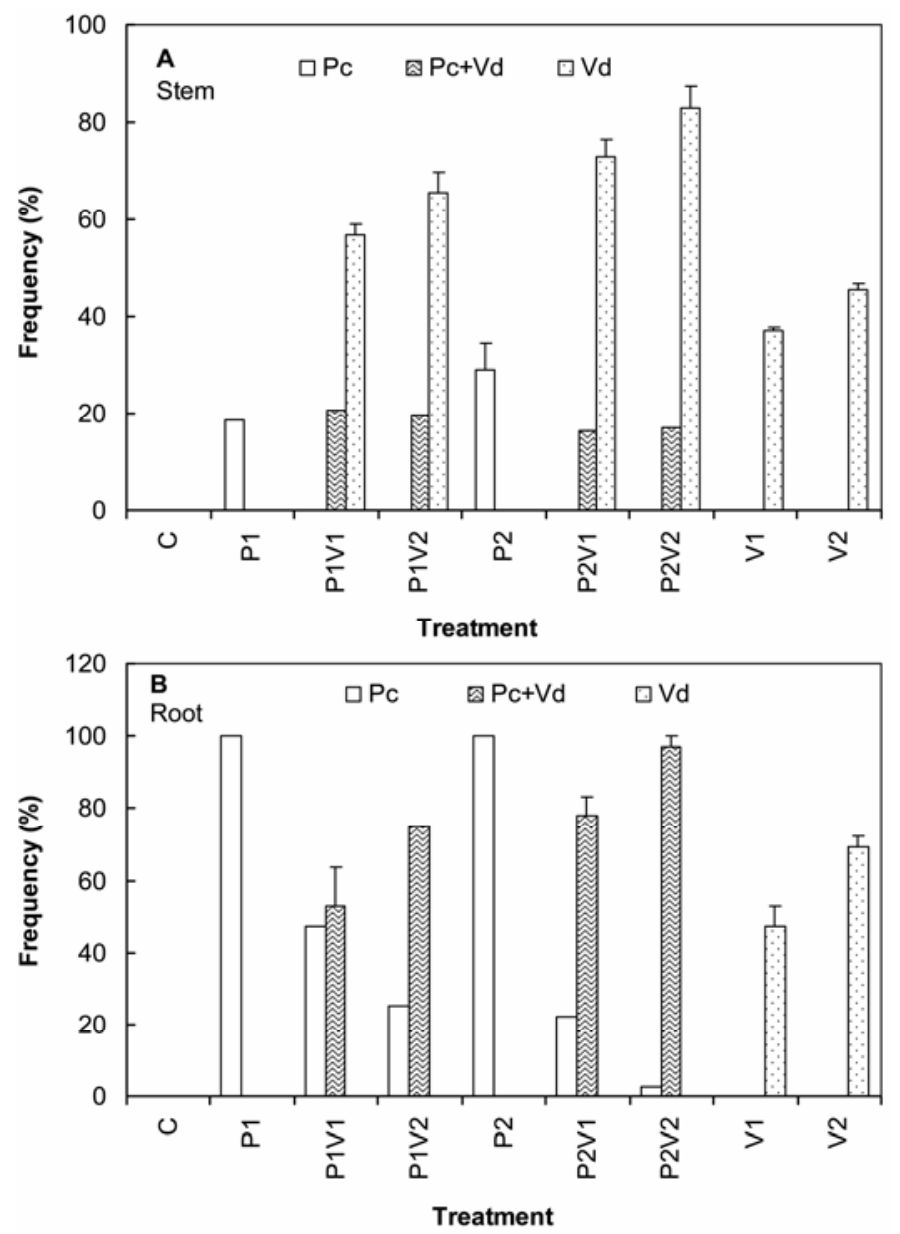

Fig. 1. Frequency of isolation of Phytophthora capsici and Verticillium dahliae from $\mathbf{A}$, stems and $\mathbf{B}$, roots of chile pepper inoculated with each pathogen alone or inoculated simultaneously with both pathogens. The abbreviations $\mathrm{Pc}, \mathrm{Vd}$, and $\mathrm{Pc}+\mathrm{Vd}$ denote isolation frequency of $P$. capsici alone, $V$. dahliae alone, and concomitant isolation of $P$. capsici and $V$. dahliae, respectively. Treatments consist of two inoculum levels of $P$. capsici $(\mathrm{P} 1=$ low and $\mathrm{P} 2=$ high), two inoculum levels of $V$. dahliae $(\mathrm{V} 1=$ low and $\mathrm{V} 2=$ high$)$, four combinations of both pathogens at each of the two inoculum levels (P1V1, P1V2, P2V1, and P2V2), and a control (C) treatment (noninoculated). Each column is the mean of three individual experiments comprising three replicates per treatment. The bar on each column represents the standard error of the mean. Bars that are too small are not visible.
There was a significant difference among treatments $\left(P<10^{-5}\right)$ as determined by Kruskal-Wallis test (Table 2). Whether plants were inoculated with $P$. capsici prior to inoculation with $V$. dahliae or vice versa, the RTE were comparable. The RTE for the two inoculum levels of $V$. dahliae used alone (V1 and V2) were identical to that of the control treatment, with an RTE $=0.115$ (Table 2). When plants were inoculated with $P$. capsici before inoculation with $V$. dahliae, RTE values were similar at 0.624 at both low (V1) and high (V2) inoculum levels of $V$. dahliae when $P$. capsici was used at a low (P1) inoculum level. Similarly, when $P$. capsici was used at a high (P2) inoculum level, RTE were 0.746 and 0.773 at low (V1) and high (V2) inoculum levels of $V$. dahliae, respectively. As indicated by the overlapping $95 \%$ confidence intervals, the RTE were similar between the two inoculum levels of $V$. dahliae (V1 and V2) at both inoculum levels of $P$. capsici ( $\mathrm{P} 1$ or $\mathrm{P} 2)$.

When plants were inoculated with $V$. dahliae before inoculation with $P$. capsici, RTE values were 0.577 and 0.652 at low (P1) and high (P2) inoculum levels of $P$. capsici, respectively, when $V$. dahliae was used at a low inoculum level (V1). Similarly, when $V$. dahliae was used at a high inoculum level (V2), RTE values were 0.605 and 0.746 at low (P1) and high (P2) inoculum levels of $P$. capsici, respectively. As indicated by the overlapping $95 \%$ confidence intervals, RTE values were similar between the two inoculum levels of $V$. dahliae (V1 and V2) at both inoculum levels of $P$. capsici (P1 or $\mathrm{P} 2)$.

Frequency of recovery of $P$. capsici from stem segments inoculated with $P$. capsici alone was $\approx 24$ and $29 \%$ at low (P1) and high (P2) inoculum levels of $P$. capsici, respectively (Fig. 2). In plants inoculated with both $P$. capsici and $V$. dahliae, $P$. capsici never was recovered alone, but always was recovered along with $V$. dahliae, and the levels of recovery were comparable with those from plants inoculated by $P$. capsici alone. Both $P$. capsici and $V$. dahliae were concomitantly recovered from the stem, and recovery was restricted to segments with necrosis in the lower stem area. The frequencies of isolation of both pathogens ranged from

TABLE 2. Median, mean rank, relative treatment effects (RTE), and 95\% confidence intervals (CI) for RTE of severity ratings on chile pepper (cv. Joe Parker) inoculated sequentially with Phytophthora capsici or Verticillium dahliae $^{\mathrm{a}}$

\begin{tabular}{lcccc}
\hline Treatment $^{\mathrm{b}}$ & Median $^{\mathrm{c}}$ & Mean rank & RTE & 95\% CI for RTE \\
\hline C & 0 & 9.5 & 0.115 & $(0.115,0.115)$ \\
P1 & 2 & 32 & 0.404 & $(0.295,0.512)$ \\
P1BV1 & 3.5 & 49.2 & 0.624 & $(0.457,0.791)$ \\
P1BV2 & 3.5 & 49.2 & 0.624 & $(0.457,0.791)$ \\
P2 & 2 & 32 & 0.404 & $(0.295,0.512)$ \\
P2BV1 & 4 & 58.6 & 0.746 & $(0.606,0.885)$ \\
P2BV2 & 4 & 60.8 & 0.773 & $(0.682,0.865)$ \\
V1 & 0 & 9.5 & 0.115 & $(0.115,0.115)$ \\
V1BP1 & 3 & 45.5 & 0.577 & $(0.424,0.730)$ \\
V1BP2 & 3.5 & 51.3 & 0.652 & $(0.505,0.800)$ \\
V2 & 0 & 9.5 & 0.115 & $(0.115,0.115)$ \\
V2BP1 & 3 & 47.6 & 0.605 & $(0.467,0.742)$ \\
V2BP2 & 4 & 58.6 & 0.746 & $(0.606,0.885)$ \\
\hline
\end{tabular}

${ }^{a}$ Kruskal-Wallis test: test statistic $=55.66, P<10^{-5}$

b Treatments consisted of two inoculum levels of $P$. capsici $(\mathrm{P} 1=$ low and $\mathrm{P} 2=$ high), two inoculum levels of $V$. dahliae $(\mathrm{V} 1=$ low and $\mathrm{V} 2=$ high $)$, eight combinations of both pathogens at each of the two inoculum levels (P1BV1, P1BV2, P2BV1, P2BV2, V1BP1, V2BP1, V1BP2, and V2BP2), and a control (C) treatment (noninoculated). The letter "B" signifies "before" and indicates which pathogen and inoculum level were used first in the sequential inoculations. For example, inoculation of plants with $P$. capsici at a low inoculum level (P1) 4 days before inoculation with $V$. dahliae at a low inoculum level (V1) is denoted as P1BV1. Similarly, inoculation of plants with $V$. dahliae at a low inoculum level (V1) 4 days before inoculation with $P$. capsici at a low inoculum level (P1) is denoted as V1BP1.

${ }^{c}$ Disease severity was rated using the following scale: $0=$ no visual disease symptom, 1 = vascular discoloration or stem necrosis, 2 = vascular discoloration and stem necrosis, $3=$ wilting and no vascular discoloration, $4=$ wilting and vascular discoloration, and $5=$ dead. 
18 to $25 \%$ across the combination of inoculum levels (P1BV1, P1BV2, P2BV1, P2BV2, V1BP1, V2BP1, V1BP2, and V2BP2) of both pathogens, and there was no significant difference among these treatments as assessed by linear contrast among treatments $(P>0.10)$.

Frequency of isolation of $V$. dahliae from stem segments was $\approx 41$ and $44 \%$ in plants inoculated with $V$. dahliae alone at low (V1) and high (V2) inoculum levels of $V$. dahliae, respectively (Fig. 2). In plants inoculated with $P$. capsici prior to inoculation with $V$. dahliae, the frequency of isolation of $V$. dahliae was $\approx 65$ and $74 \%$ at low (V1) and high (V2) inoculum levels of V. dahliae, respectively, when $P$. capsici was used at a low (P1) inoculum level. The frequency of isolation of $V$. dahliae was $\approx 79 \%$ at both low (V1) and high (V2) inoculum levels of $V$. dahliae, respectively, when $P$. capsici was used at a high inoculum level (P2). These frequencies of recovery of $V$. dahliae from stem segments in the presence of $P$. capsici were significantly higher, by 59 to $90 \%$, than the frequency of its recovery when plants were inoculated with $V$. dahliae alone, as revealed by linear contrasts $(P<0.0001)$.

In plants inoculated with $V$. dahliae prior to inoculation with $P$. capsici, the frequency of isolation of $V$. dahliae was 71 and $78 \%$ at low (P1) and high (P2) inoculum levels of P. capsici, respectively, when $V$. dahliae was used at a low (V1) inoculum level (Fig. 2). Similarly, when $V$. dahliae was used at a high inoculum level (V2), the frequency of isolation of $V$. dahliae was 75 and $81 \%$ at low (P1) and high (P2) inoculum levels of $P$. capsici, respectively. These frequencies of recovery of $V$. dahliae from stem segments in the presence of $P$. capsici were significantly higher, by 70 to $90 \%$, than the frequency of its recovery when plants were inoculated with $V$. dahliae alone $(P<0.0001)$.

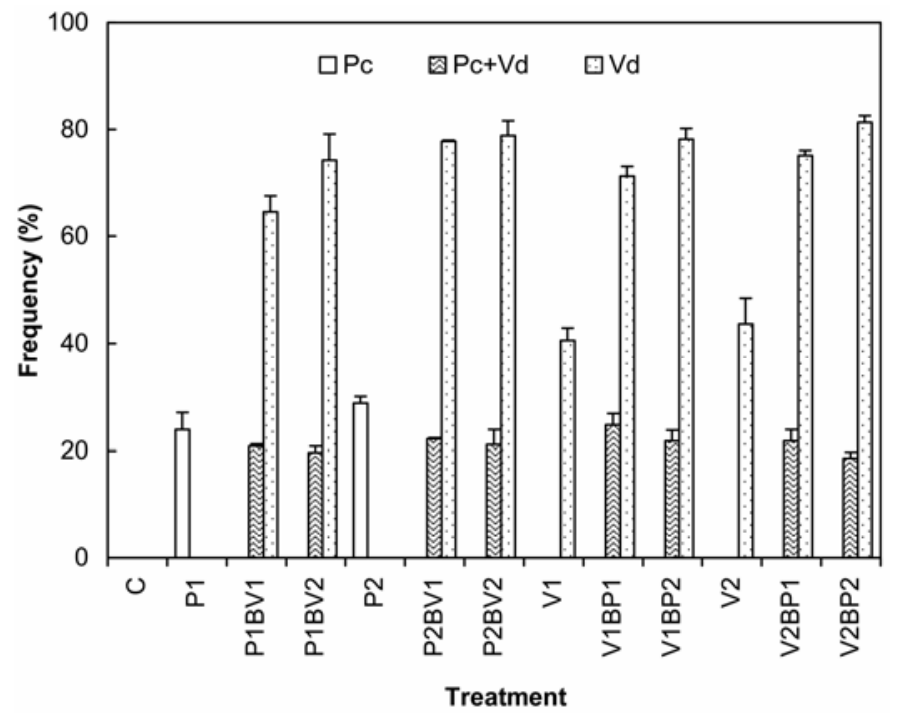

Fig. 2. Frequency of isolation of Phytophthora capsici and Verticillium dahliae from stems of chile pepper inoculated with each pathogen alone or inoculated sequentially with both pathogens. The abbreviations $\mathrm{Pc}, \mathrm{Vd}$, and $\mathrm{Pc}+\mathrm{Vd}$ denote isolation frequency of $P$. capsici alone, $V$. dahliae alone, and concomitant isolation of $P$. capsici and $V$. dahliae, respectively. Treatments consist of two inoculum levels of $P$. capsici $(\mathrm{P} 1=$ low and $\mathrm{P} 2=$ high $)$, two inoculum levels of $V$. dahliae $(\mathrm{V} 1=$ low and $\mathrm{V} 2=$ high$)$, eight combinations of both pathogens at each of the two inoculum levels (P1BV1, P1BV2, P2BV1, $\mathrm{P} 2 \mathrm{BV} 2$, V1BP1, V2BP1, V1BP2, and V2BP2), and a control (C) treatment (noninoculated). The letter "B" signifies "before" and indicates which pathogen and inoculum level were used first in the sequential inoculations. For example, inoculation of plants with P. capsici at a low inoculum level (P1) 4 days before inoculation with $V$. dahliae at a low inoculum level (V1) is denoted as P1BV1. Similarly, inoculation of plants with $V$. dahliae at a low inoculum level (V1) 4 days before inoculation with $P$. capsici at a low inoculum level (P1) is denoted as V1BP1. Each column is the mean of two individual experiments comprising three replicates per treatment. The bar on each column represents the standard error of the mean. Bars that are too small are not visible.
Whether plants were inoculated with $P$. capsici prior to inoculation with $V$. dahliae or vice versa, there was a comparable increase in the recovery of $V$. dahliae from stems under both scenarios. Taking into account the levels of concomitant recovery (stem segments with both $V$. dahliae and $P$. capsici), the frequency of recovery of $V$. dahliae was higher, by 109 to $144 \%$, than the frequency of its recovery when plants were inoculated with V. dahliae alone.

Pathogen interaction on growth media. In simultaneous pairing of $P$. capsici and $V$. dahliae, mycelial growth of $P$. capsici grown alone $(\mathrm{P})$ was not significantly different from mycelial growth of $P$. capsici grown together with $V$. dahliae $(\mathrm{vP})$ across all media $(P>0.1)$. Similarly, mycelial growth of $V$. dahliae grown alone $(\mathrm{V})$ was not significantly different from mycelial growth of $V$. dahliae grown together with $P$. capsici $(\mathrm{Vp})$ across all media $(P>0.08)$. These results were consistent in both pairing time periods ( $72 \mathrm{~h}$ and 7 days) across all growth media (Fig. 3A and B). Upon contact, which occurred between 72 and 84 h, mycelia
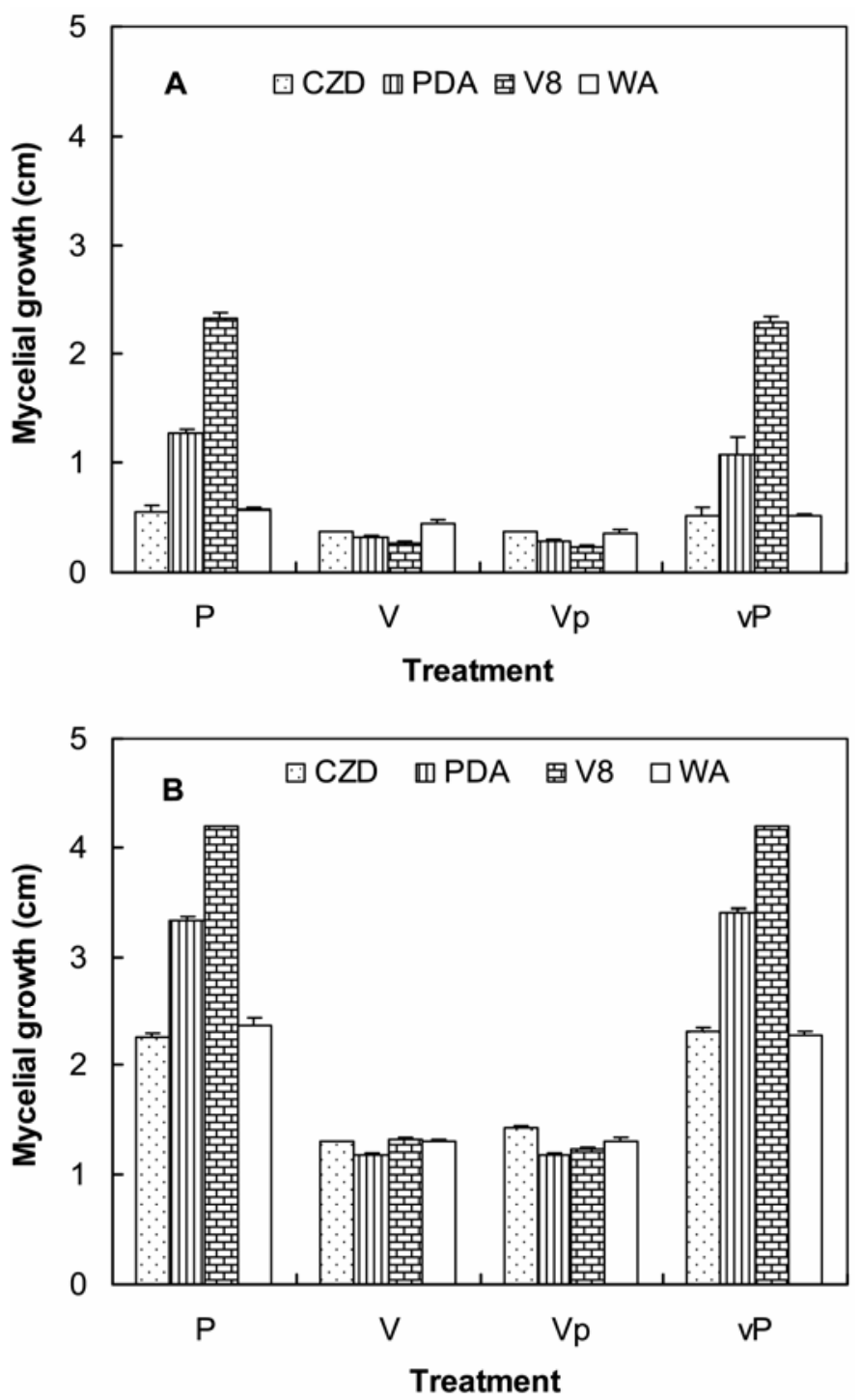

Fig. 3. Radial growth of Phytophthora capsici and Verticillium dahliae grown alone or paired on various growth media for $\mathbf{A}, 72 \mathrm{~h}$ and $\mathbf{B}, 7$ days. The abbreviations V8, PDA, CZD, and WA denote V8 juice agar, potato dextrose agar, Czapek-Dox agar, and water agar media, respectively. The designations $\mathrm{P}, \mathrm{vP}, \mathrm{V}$, and $\mathrm{Vp}$ represent $P$. capsici grown alone, $P$. capsici grown with $V$. dahliae, V. dahliae grown alone, and V. dahliae grown with $P$. capsici, respectively. Each column is the mean of three individual experiments comprising five or six replicates per treatment. The bar on each column represents the standard error of the mean. Bars that are too small are not visible. 
of $P$. capsici and $V$. dahliae intermingled with no indication of inhibition. By 7 days, P. capsici totally overgrew V. dahliae on V8 juice agar and potato dextrose agar media; however, the edges of $V$. dahliae colonies were clearly visible within the colonies of $P$. capsici. In contrast, on water agar and Czapek-Dox media, $P$. capsici overgrew $V$. dahliae colonies by 30 to $50 \%$.

When $P$. capsici and $V$. dahliae were paired, 7 days after initial establishment of $V$. dahliae (Fig. 4), mycelial growth of $\mathrm{P}$ was not significantly different from mycelial growth of vP across all media $(P>0.1)$. Similarly, mycelial growth of $\mathrm{V}$ was not significantly different from mycelial growth of $\mathrm{Vp}$ across all media $(P>0.1)$.

Under conditions which were designed to test for production and activity of volatile substances using inverted culture plates (Fig. 5), mycelial growth of $\mathrm{P}$ was not significantly different from mycelial growth of vP across all media $(P>0.07)$. Similarly, mycelial growth of $\mathrm{V}$ was not significantly different from mycelial growth of $\mathrm{Vp}$ across all media $(P>0.4)$.

\section{DISCUSSION}

Interactions between $P$. capsici and $V$. dahliae have not been reported previously. This is the first known report focusing on the interactive effects of $P$. capsici and $V$. dahliae on chile pepper. The salient information emanating from this study indicates that the presence of $P$. capsici and $V$. dahliae in the same inoculum court enhances infection and colonization of chile pepper by $V$. dahliae. The notion of plant infection and colonization by one pathogen being facilitated by another pathogen has been well established in many other pathosystems $(5,8,12,13)$. However, this notion is new in the case of chile pepper infection by $P$. capsici and $V$. dahliae. Consequently, no other studies are available for comparison of results.

The methodological approaches used were similar to those used by others who have examined interactions among other mycelial microorganisms in vitro and in planta $(5,8,12,13,18,20)$. The in

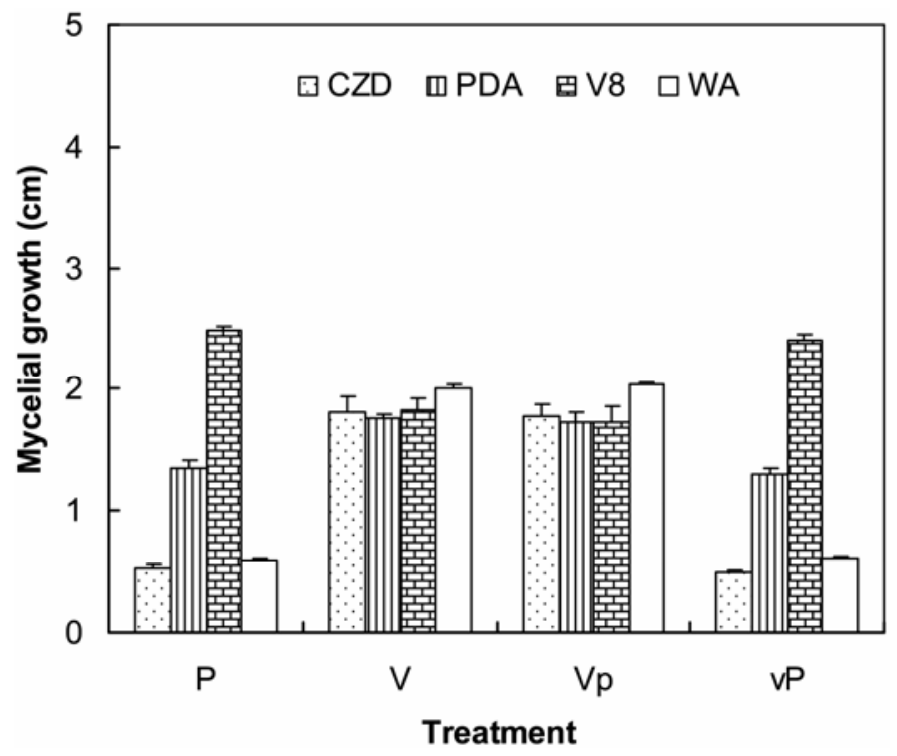

Fig. 4. Radial growth of Phytophthora capsici and Verticillium dahliae grown alone or paired on various growth media when $P$. capsici establishment was delayed 7 days. Radial growth was measured $72 \mathrm{~h}$ following pairing of $V$. dahliae with $P$. capsici. The abbreviations V8, PDA, CZD, and WA denote V8 juice agar, potato dextrose agar, Czapek-Dox agar, and water agar media, respectively. The designations $\mathrm{P}, \mathrm{vP}, \mathrm{V}$, and $\mathrm{Vp}$ represent $P$. capsici grown alone, $P$. capsici grown with $V$. dahliae, $V$. dahliae grown alone, and $V$. dahliae grown with $P$. capsici, respectively. Each column is the mean of three individual experiments comprising five or six replicates per treatment. The bar on each column represents the standard error of the mean. Bars that are too small are not visible. vitro studies on growth media were designed to provide a benchmark evaluation of interactions between $P$. capsici and $V$. dahliae under various nutritional environments as represented by the different growth media. Although these media are not comparable with plant tissue, it was assumed that pathogenic interactions could take place in plant tissue of varying nutritional status. Therefore, it was important to ascertain whether pathogenic interactions were influenced under different nutritional environments. Gauged on the consistency of the responses of $P$. capsici and $V$. dahliae across all growth media used, it appears from this study that nutritional environments may not exert a significant effect on the interaction between $P$. capsici and $V$. dahliae.

Outcomes of studies that addressed mycelial interactions among microorganisms have been cataloged by Stahl and Christensen (18) into four types: invasion-lysis, invasion-overgrowth, contact inhibition, and inhibition at a distance. $P$. capsici grew extensively and overgrew colonies of $V$. dahliae on all media. At points of contact, colonies intermingled with no sign of inhibition. Therefore, the interaction between $P$. capsici and $V$. dahliae is of an invasion-overgrowth type following descriptions by Stahl and Christensen (18).

For each pathogen, the levels of inoculum used were selected from previous studies $(3,15)$. Although it is not known whether these levels are comparable with those encountered in chile pepper fields, symptom development under the selected levels mimicked well that under field conditions as observed in previous experiments for seedling screening for disease resistance and pathogen population characterization $(3,15)$. The phenological stage used in this study was selected to represent the stage at which disease onset is observed in production fields. Typically, wilt onset is observed beginning at approximately flowering and onward through fruit set, maturation, and ripening.

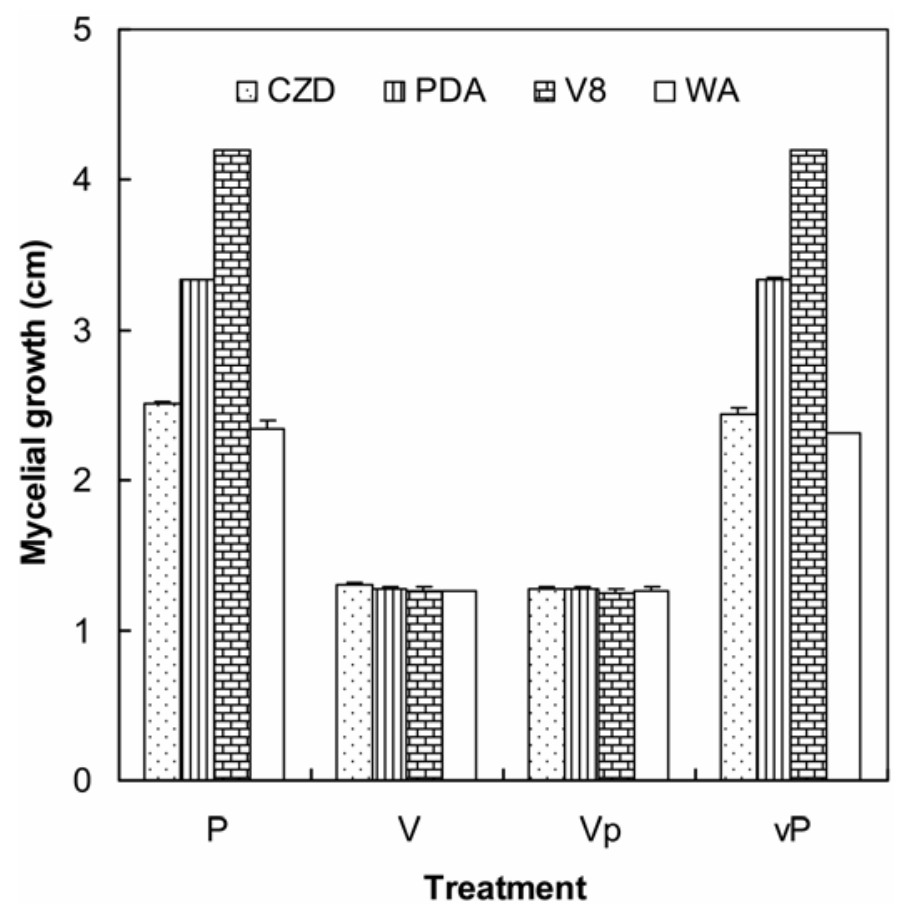

Fig. 5. Radial growth of Phytophthora capsici and Verticillium dahliae grown alone or together for 7 days on various growth media in inverted culture plate environment to test for production and activity of volatile substances. The abbreviations V8, PDA, CZD, and WA denote V8 juice agar, potato dextrose agar, Czapek-Dox agar, and water agar media, respectively. The designations $\mathrm{P}$, vP, V, and $\mathrm{Vp}$ represent $P$. capsici grown alone, $P$. capsici grown with $V$. dahliae, V. dahliae grown alone, and V. dahliae grown with $P$. capsici, respectively. Each column is the mean of three individual experiments comprising six replicates per treatment. The bar on each column represents the standard error of the mean. Bars that are too small are not visible. 
The three sets of experiments conducted on plant infection were designed to represent three scenarios of plant infection by $P$. capsici and $V$. dahliae: simultaneous infection, or sequential infection in which $P$. capsici infection precedes $V$. dahliae infection or vice versa. It is not known which of these scenarios would be considered as the norm under field conditions. Nonetheless, this study has delineated an outcome common to all three scenarios, which is that of a higher infection level by $V$. dahliae in the presence of $P$. capsici than when plants were inoculated with $V$. dahliae alone. In the sequential inoculations, when plants were inoculated with $P$. capsici 4 days prior to inoculation with $V$. dahliae or vice versa, the period of 4 days was chosen because symptoms of plant infection by $P$. capsici were reported at and beyond 4 days of inoculation (1). It was assumed that the 4-day period was sufficient for plant infection by $P$. capsici or $V$. dahliae. $P$. capsici is a strong plant colonizer and probably was the first to invade the root and paved the way for subsequent increased colonization by $V$. dahliae. In this study, $P$. capsici acts as a facilitator for $V$. dahliae. Similar results were reported by others. For example, in peanut (Arachis hypogaea), Frank (8) found that Fusarium solani predisposed pods to increased rotting activities by Pythium myriotylum.

Studies on interactions between microorganisms with differential infection patterns may be faced with several experimental issues, one of which is defining the time for assessing plant infection. Both pathogens can infect plants at any time, but differ in terms of incubation period, a variable that is influenced by host plant, pathogen, and environmental factors (13). Under controlled-environment conditions of greenhouse or growth chamber, symptoms of infection of pepper by $P$. capsici have been observed as early as 4 days (1) to over 7 days (17) after inoculation. In contrast, symptoms of pepper infection by $V$. dahliae were reported within 3 weeks $(7,10)$ to over 6 weeks $(13,17)$ after inoculation. In this study, assessment of interaction between $P$. capsici and $V$. dahliae was made at 21 days after inoculation with $P$. capsici for two reasons. First, preliminary work showed that all plants inoculated with $P$. capsici died beyond 21 days. Second, plant colonization by $V$. dahliae as gauged by development of $V$. dahliae colonies from stem segments was shown to occur within 21 days of inoculation (10) and is corroborated by the results reported herein.

There is an indication that $P$. capsici and $V$. dahliae act synergistically if incubation period and RTE are taken into account. Symptoms of wilting first were observed in plants inoculated with both $P$. capsici and $V$. dahliae. In addition, RTE generally was greater in plants inoculated with both pathogens than in plants inoculated with each pathogen separately. It is concluded that, under condition of coexistence, $P$. capsici and $V$. dahliae may hasten the development of wilt on chile pepper, as previously postulated (17).

The significance of this work is two-pronged: (i) it provides an advanced understanding of the interaction between $P$. capsici and $V$. dahliae, two pathogens with differential infection patterns; and (ii) it demonstrates the plausibility of an increased incidence and hastened development of wilt symptoms in chile pepper under conditions of coexistence of $P$. capsici and $V$. dahliae in the same field. The findings from this study suggest that $P$. capsici acts as a co-factor in infection and colonization of chile pepper by $V$. dahliae. Therefore, in fields infested by P. capsici and V. dahliae, control measures should be focused on both microorganisms.

\section{ACKNOWLEDGMENTS}

Financial support for this research was provided by the New Mexico Agricultural Experiment Station. I thank C. Sanogo and S. Thomas for reviewing this manuscript.

\section{LITERATURE CITED}

1. Aguirreolea, J., Irigoyen, J, Sánchez-Díaz, M., and Salaverri, J. 1995. Physiological alterations in pepper during wilt induced by Phytophthora capsici and soil water deficit. Plant Pathol. 44:587-596.

2. Biles, C., Lindsey, D. L., and Liddell, C. M. 1992. Control of Phytophthora root rot of chile peppers by irrigation practices and fungicides. Crop Prot. 11:225-228.

3. Bosland, P. W., and Lindsey, D. L. 1991. A seedling screen for Phytophthora root rot of pepper, Capsicum annuum. Plant Dis. 75:1048-1050.

4. Brunner, E., Domhof, S., and Langer, F. 2002. Nonparametric Analysis of Longitudinal Data in Factorial Experiments. John Wiley \& Sons, New York.

5. Davis, J. R., and Howard, M. N. 1976. Presence of Colletotrichum atramentarium in Idaho and relation to Verticillium wilt (Verticillium dahliae). (Abstr.) Am. Potato J. 53:397-398.

6. Erwin, D. C., and Ribeiro, O. K. 1996. Phytophthora Diseases Worldwide. The American Phytopathological Society, St. Paul, MN.

7. Evans, G., and McKeen, C. D. 1975. A strain of Verticillium dahliae pathogenic to sweet pepper in southwestern Ontario. Can. J. Plant Sci. 55:857-859.

8. Frank, Z. R. 1972. Pythium myriotylum and Fusarium solani as cofactors in a pod-rot complex of peanut. Phytopathology 62:1331-1334.

9. Goicoechea, N., Aguirreola, J., Cenoz, S., and Garcia-Mina, J. M. 2000. Verticillium dahliae modifies the concentrations of proline, soluble sugars, starch, soluble protein and abscisic acid in pepper plants. Eur. J. Plant Pathol. 106:19-25.

10. Goicoechea, N., Aguirreola, J., Cenoz, S., and Garcia-Mina, J. M. 2001. Gas exchange and flowering in Verticillium-wilted pepper plants. J. Phytopathol. 149:281-286.

11. Gomez, K. A., and Gomez, A. A.1984. Statistical Procedures for Agricultural Research, 2nd ed. John Wiley \& Sons, Inc., New York.

12. Kotcon, J. B., Rouse, D. I., and Mitchell, J. E. 1985. Interactions of Verticillium dahliae, Colletotrichum coccodes, Rhizoctonia solani, and Pratylenchus penetrans in the early dying syndrome of Russet Burbank potatoes. Phytopathology 75:68-74.

13. Otazu, V., Gudmestad, N. C., and Zink, R. T. 1978. The role of Colletotrichum atramentarium in the potato wilt complex in North Dakota. Plant Dis. Rep. 62:847-851.

14. Pegg, G. F., and Brady, B. L. 2002. Verticillium Wilts. CABI Publishing, CAB International, Wallingford, UK.

15. Riggs, J. L., and Graham, C. J. 1995. A screening of New Mexico Verticillium dahliae isolates for cross-infectivity to cotton and chile. Pages 218-221 in: Proc. 1995 Beltwide Cotton Conference. National Cotton Council, Memphis, TN.

16. Sanogo, S. 2003. Chile pepper and the threat of wilt diseases. Online. Plant Health Progress doi:10.1094/PHP-2003-0430-01-RV.

17. Sanogo, S., and Carpenter, J. 2006. Incidence of Phytophthora blight and Verticillium wilt within chile pepper fields in New Mexico. Plant Dis. 90:291-296.

18. Stahl, P. D., and Christensen, M. 1992. In vitro mycelial interactions among members of a soil microfungal community. Soil Biol. Biochem. 24:309-316.

19. Tomescu, A., Voican, V., and Racaru, V. 2000. Some physiological changes induced by the fungus Verticillium dahliae in pepper plants. Pages 151-154 in: Advances in Verticillium Research and Disease Management. E. C. Tjamos, R. C. Rowe, J. B. Heale, and D. R. Fravel, eds. The American Phytopathological Society, St. Paul, MN.

20. Whipps, J. M. 1987. Effect of media on growth and interactions between a range of soil-borne glasshouse pathogens and antagonistic fungi. New Phytol. 107:127-142.

21. Xie, J., Cardenas, E. S., Sammis, T. W., Wall, M. M., Lindsey, D. L., and Murray, L. W. 1999. Effects of irrigation method on chile pepper yield and Phytophthora root rot incidence. Agric. Water Manage. 42:127-142. 